\title{
Colorectal Cancer Awareness and Screening Preference: A Survey during the Malaysian World Digestive Day Campaign
}

\author{
Mohd Azri Mohd Suan'*, Noor Syahireen Mohammed ${ }^{1}$, Muhammad Radzi Abu \\ Hassan $^{2}$
}

\begin{abstract}
Background: Although the incidence of colorectal cancer in Malaysia is increasing, awareness of this cancer, including its symptoms, risk factors and screening methods, remains low among Malaysian populations. This survey was conducted with the aim of (i) ascertaining the awareness level regarding colorectal cancer symptoms, risk factors and its screening among the general populations and (ii) assessing the public preference and willingness to pay for colorectal cancer screening. Materials and Methods: The questionnaire was distributed in eight major cities in West Malaysia during the World Health Digestive Day (WDHD) campaign. Two thousand four hundred and eight respondents participated in this survey. Results: Generally, awareness of colorectal cancer was found to be relatively good. Symptoms such as change in bowel habit, blood in the stool, weight loss and abdominal pain were well recognized by $86.6 \%, 86.9 \%, 83.4 \%$ and $85.6 \%$ of the respondents, respectively. However, common risk factors such as positive family history, obesity and old age were acknowledged only by less than $\mathbf{7 0 \%}$ of the respondents. Almost $80 \%$ of the respondents are willing to take the screening test even without any apparent symptoms. Colonoscopy is the preferred screening method, but only $37.5 \%$ were willing to pay from their own pocket to get early colonoscopy. Conclusions: Continous cancer education should be promoted with more involvement from healthcare providers in order to make future colorectal cancer screening programs successful.
\end{abstract}

Keywords: Awareness - colonoscopy - colorectal cancer - Malaysia - screening

Asian Pac J Cancer Prev, 16 (18), 8345-8349

\section{Introduction}

Colorectal cancer is common in Malaysia, both in men and women. In restropect, incidence of colorectal cancers in Malaysia has been reported in the international journal since 1965 (Lim, 2001). According to the Malaysian National Cancer Registry, in year 2003, colorectal cancer is the top and third most frequently reported cancer in both males and females respectively, in West Malaysia (Lim et al., 2004). More recently, report from the National Cancer Patient Registry-Colorectal Cancer stated that from year 2008 to 2013, overall incidence rate for colorectal cancer was 21.3 cases per 100, 000 populations in Malaysia (Radzi et al., 2014). More worrying was the fact that the overall mortality rate was 9.8 cases per 100,000 populations during the same period of time with men having higher mortality rate than women.

Despite the increasing incidence of colorectal cancer, awareness on colorectal cancer, including its symptoms, risk factors and screening method, remains low in Malaysian populations. Hashim et al. (2011) conducted a survey on knowledge about colorectal cancer among patients presented with rectal bleeding in a Malaysian university hospital. They found that majority of surveyed patients were unable to identify symptoms of colorectal cancer except for rectal bleeding. Knowledge on risk factors for the same cancer was also found to be low. In another study by Al-Naggar and Bobryshev (2013), a large number of university students in Malaysia did not know about any specific methods for colorectal cancer screening. Furthermore, a study by Harmy et al. (2012) reported only $7 \%$ from nearly 2000 healthy respondent were aware of colorectal cancer screening. Apprehension and embarrassment were found to be the primary reasons for not participating in the screening.

Since there is no population-based screening program available for colorectal cancer in Malaysia, it is unknown as to what extent the Malaysian populations would be receptive to colorectal cancer screening. The information on preferred colorectal cancer screening tools among Malaysian populations needs to be identified if the screening uptake is to be increased. The primary aim of this study is to ascertain the awareness level towards colorectal cancer symptoms, risk factors and its screening among general populations in Malaysia. Secondly, the authors would like to assess the public preference and willingness to pay for colorectal cancer screening.

${ }^{I}$ Clinical Research Center, ${ }^{2}$ Department of Internal Medicine, Sultanah Bahiyah Hospital, Alor Setar, Kedah, Malaysia *For correspondence:irzah96@yahoo.com 


\section{Materials and Methods}

This is a prospective cross sectional study using a selfadministered questionnaire to collect data of interest. The questionnaire was adapted from Harewood et al. (2009), and sought information regarding;

Respondents' demographic

Awareness on cancer statistic

Awareness of colorectal cancer symptoms and risk factors

Understanding of the concept of screening

Preferred method of screening test

Willingness to pay for colonoscopy as a screening tool

The questionnaire comprised of 22 questions and took

Table 1. Socio-demographic Characteristics of Respondents

\begin{tabular}{lrc}
\hline Characteristics & $\mathrm{n}$ & $(\%)$ \\
\hline Gender & & \\
$\quad$ Female & 1366 & 56.7 \\
$\quad$ Male & 1042 & 43.3 \\
Age, years old & & \\
$\quad$ Less than 50 & 2111 & 87.7 \\
$\quad$ More than 50 & 297 & 12.3 \\
Ethnicity & & \\
$\quad$ Malay & 916 & 38 \\
$\quad$ Chinese & 1101 & 45.7 \\
Indian & 320 & 13.3 \\
$\quad$ Others & 71 & 3 \\
Level of Education & & \\
$\quad$ Primary & 136 & 5.6 \\
$\quad$ Secondary & 755 & 31.4 \\
$\quad$ Tertiary & 1412 & 58.6 \\
$\quad$ No formal education & 105 & 4.4 \\
Smoking status & & \\
$\quad$ Yes & 234 & 9.7 \\
$\quad$ No & 2163 & 89.8 \\
$\quad$ NA & 11 & 0.5 \\
\hline NA = not available & &
\end{tabular}

approximately ten minutes to complete. Permission to use the questionnaire has been obtained from the original author.

The questionnaire was distributed during the World Health Digestive Day (WDHD) campaign in Malaysia. This annual event organized by the Digestive Health Advisory Board (DHAB) to increase general public awareness of prevention and therapy towards healthy gut. Several booths were setup at various shopping centers throughout the country during the campaign period. Those who visited the campaign booth were offered to complete the study questionnaire after reading and consenting to the study information sheet. Potential respondents with known case of colorectal cancer, had first degree family members with colorectal cancer, history of familial adenosis polyposis or chronic inflammatory bowel disease were excluded. All data was collected anonymously.

Statistical analyses were performed on the data collected using SPSS software (version 20.0) and the results of the survey were presented in percentage (\%). The conduct of this study has been approved by Medical Research Ethics Committee, Ministry of Health Malaysia (NMRR-15-474-25726).

\section{Results}

Two thousand four hundred and eight respondents voluntarily answered the questionnaires that were distributed at eight different cities throughout peninsular Malaysia. In northern region of Peninsular Malaysia, the cities involved were Bayan Lepas and Georgetown in Penang and also Sungai Petani in Kedah. Cities in central region were Ipoh, Perak as well as Damansara and Petaling Jaya in Selangor. In southern states were Bandaraya Melaka in Malacca and Johor Bahru in Johor. Details of respondents' socio-demographic characteristics were presented in Table 1. More than half of the respondents were female (56.7\%) and aged less

Table 2. Respondents awareness on colorectal cancer.

\begin{tabular}{llc}
\hline Questions & Response options & Responses (n=2408), \% \\
\hline Colorectal cancer is among the top 3 cancers in Malaysia. & Agree & 87.5 \\
& Disagree & 12.5 \\
Colorectal cancer usually starts from polyps in the colon or rectum. & Agree & 87.7 \\
& Disagree & 12.3 \\
Colorectal cancer often starts without showing any symptoms. & Agree & 72.8 \\
Symptoms related to Colorectal Cancer? & Disagree & 27.2 \\
& Change in bowel habit & 86.6 \\
& Blood in the stool & 86.9 \\
Risk factor for Colorectal Cancer? & Weight loss & 83.4 \\
& Abdominal pain & 68.6 \\
& Positive family history & 59.1 \\
Screening can find cancer early when treatment can be the most effective. & Obesity & 56.4 \\
Late detection of late stage of bowel cancer carries poorest survival rate. & Old age & 72.3 \\
& Low fibre diet consumption & 77.5 \\
& Inflammatory bowel disease & 91.3 \\
\hline
\end{tabular}


Table 3. Respondents Willingness for Screening and Preferred Colorectal Cancer Screening Method

\begin{tabular}{lr}
\hline Questions & Responses $(\mathrm{n}=2408), \%$ \\
\hline Willing to have a screening test for the bowel cancer, even without any symptom? & 79.5 \\
Yes & 18.5 \\
No & 2.0 \\
No answer given & 31.6 \\
Preferred Colorectal Cancer screening method if keen for screening? & 11.5 \\
Faecal blood test & 36.5 \\
Sigmoidoscopy & 20.4 \\
Colonoscopy & 37.5 \\
Unsure & \\
Will pay RM800 (own money) and have a test & 46.5 \\
within a week, $\%$ & 13.6 \\
Will wait for 6-8 months for free test, $\%$ & 2.4 \\
Not prefer to have test at all, \% & \\
No answer given, $\%$ & \\
\hline
\end{tabular}

than 50 years old $(87.7 \%)$. Majority of the respondents were Chinese $(45.7 \%)$, followed with Malay $(38.0 \%)$, Indian (13.3\%) and other ethnicity (3.0\%). Most of the respondent received formal education with $58.6 \%$ had tertiary education. Only $4.4 \%$ of respondent reported not receiving any formal education. Almost $90 \%$ of the respondents were non-smoker. However, data on smoking status is not available for 11 respondents.

Majority of the respondents $(87.5 \%)$ were aware that colorectal cancer is among the top 3 cancers in Malaysia (Table 2). When asked about the origin of colorectal cancer, $87.7 \%$ knew that the cancer usually starts from polyps in the colon or rectum. A small proportion of the respondents $(27.2 \%)$ disagreed that colorectal cancer may develop without exhibiting any symptoms. In terms of colorectal cancer manifestations, more than $80 \%$ of respondents were aware that change in bowel habit, blood in the stool, weight loss and abdominal pain were the symptoms related to colorectal cancer. Despite this, most of respondents were surprisingly, not well aware of the risk factors for colorectal cancer. Only less than $70 \%$ of respondent can identify 'positive family history', 'obesity' and 'old age' as the risk factors for colorectal cancer.

Another notable finding was excellent public awareness towards the importance of colorectal cancer screening. A substantial number of respondents $(91.3 \%)$ agreed that screening test is able to detect the cancer early while $86.3 \%$ respondents had the same opinion that late detection of cancer carries poorer survival rate. Respondents' willingness and preferred screening test for colorectal cancer was presented in Table 3. Interestingly, almost $80 \%$ of the respondents' were willing to undergo a screening test for the bowel even though without having any symptoms. On preferred screening method, $36.5 \%$ of respondents favoured to have colonoscopy, while $31.6 \%$ and $11.5 \%$ opted for faecal occult blood test and sigmoidoscopy respectively. Respondents were also asked on their willingness to pay for colonoscopy if suggested by their physician. Less than half of the respondents $(46 \%)$ would rather choose to wait between 6-8 months to get free procedure. Only $37.5 \%$ were willing to pay from their own pocket to get early colonoscopy done whereas small proportion of respondents was unwilling to undergo colonoscopy at all.

\section{Discussion}

In order to set up an effective national colorectal cancer screening program, it is imperative to have a clear picture of what do public knows about this cancer and their preference towards the screening program. From this study, awareness among studied population on colorectal cancer was found to be relatively good. This is based on high percentage of respondents that acknowledged colorectal cancer as one of the common cancer in the country. In addition, over $70 \%$ of the respondents were aware that colorectal cancer usually starts from polyps in the bowel and can be presented without any symptoms.

This positive finding was also seen on awareness towards warning symptoms related to colorectal cancer. Symptoms such as change in bowel habit, blood in the stool, weight loss and abdominal pain were well recognized by majority of the respondents. However, this finding was conflicting with some previous studies (Hilmi et al., 2010; Loh et al., 2013; Wong et al., 2013). Loh et al. (2013) in their community-based study carried out in five rural/suburban areas in which their findings showed that awareness of warning signs for colorectal cancer were poor. Most of their study respondents were only able to identify 2 or 3 of the nine warning signs listed. This stark difference was probably due to the fact that current study conducted in urban areas where health related information was easily available and accessible. Most of the health promotion campaigns were held in major cities, hence contributed to a better awareness among urban population. Perhaps, future awareness activities need to be targeted more to the rural / suburban areas to boost public awareness.

Despite high awareness on colorectal cancer symptoms, awareness regarding risk factors associated with colorectal cancer remains low. Common risk factor such as positive family history, obesity and old age were cited by less than $70 \%$ of the respondents. This finding is in line with other local studies which found much lower 
awareness level in their respective cohort (Hilmi et al. 2010; Su et al. 2013). The above-mentioned risk factors were known for positive association with the colorectal cancer. A study from Taiwan found an increased risk for advanced cancer among immediate family members of colorectal cancer patient who has been screened (Tung and $\mathrm{Wu}, 2000)$. Obesity was found to increase the risk of colorectal cancer. Ma et al. had conducted a systematic review on 54 studies assessing the association of obesity with the risk of colorectal cancer (Ma et al., 2013). They concluded that both of general obesity (measured using Body Mass Index) and central obesity (measured using waist circumference) were highly linked to the colorectal cancer. Colorectal Cancer incidence also strongly related to age. In the United Kingdom, over $70 \%$ of newly diagnosed colorectal cancer cases were among those aged 65 and older (Office for National Statistic, 2014). Recently, local cancer registry observed similar trend in which more than $80 \%$ of colorectal cancer occur in people aged 50 or older (Radzi et al. 2014). The same registry also documented the proportion of colorectal cancer increased with age, which peak between 60 and 64 for men and between 55 and 59 for women. Consequently, failure to acknowledge these common risk factors could hamper the turn up for future colorectal cancer screening program. One of the solutions is to engage the primary care doctors and family physician in identifying and recommending high risk patients for colorectal cancer screening. The effectiveness of the family doctor's role has been proven in previous studies (Weller et al., 2009; Hewitson et al., 2011) and should be the way forward to increase awareness and cancer screening uptake.

Many studies found that positive family history of colorectal cancer as a motivational factor to undergo cancer screening test (Hlayaty et al., 2005; DelgadoPlasencia et al., 2009). Interestingly, though current studied population did not have any first degree relatives diagnosed with colorectal cancer but yet almost $80 \%$ are willing to take the screening test even without any apparent symptoms. Such positive attitude indicates that the studied population are ready and willing to partake in future colorectal cancer screening program. Despite its invasive procedure, surprisingly, colonoscopy was a preferred tool for colorectal cancer screening, followed by faecal occult blood test whereas sigmoidoscopy was the least preferred method. The above-mentioned screening techniques were recommended option for colorectal cancer screening in Asia (Sung et al., 2015). Colonoscopy for example, is regarded as the gold standard technique as it is able to visualize the entire bowel and at the same time allowing removal of any visualized pre-cancerous lesion. However, this procedure is not feasible as a mass screening technique owing to its invasive nature, requiring skilful operator, limited availability and costly. In addition, only $37.4 \%$ of respondents willing to pay to get colonoscopy done while another $46.7 \%$ respondents were rather wait for several months and get it done freely later from government hospital. Thus, the appropriate use of colonoscopy is essential to maximise the screening rate. Based on the above results, the authors suggest that colonoscopy should be prioritized to the high risk group population, especially those with positive family history and older age group. Tan et al. also suggested that priority given to patient presented with rectal bleeding and iron deficiency anemia (Tan et al., 2002). Simultaneously, concerted effort is needed to increase numbers of skill operators and availability of the procedure throughout the country. In certain Europe countries, nurses have been trained to perform endoscopy to reduce patient's waiting time (Singh et al., 2014; Joseph et al., 2015). On the other hand, faecal occult blood test can be utilized for mass screening among low risk or asymptomatic patients.

Certain limitation of this study should be noted. Respondents in current study were mostly from big city, thus the results of the present study are more representative of urban rather than a rural population. They may be differences in terms of level of awareness and willingness to participate in cancer screening program between the two populations owing to limited access to health information and services as mentioned earlier. Other than that, the respondents in this study were also been exposed to the information and materials that promotes awareness and knowledge on colorectal cancer during the campaign period. Nonetheless, this study was a community-based study, which can be perceived as more generalized than those conducted in clinics or hospitals. Thus, the results from this study should be considered vital when considering the initiation of a national colorectal cancer screening program in near future. Secondly, although the survey questions were adapted from other study (Harewood et al., 2009), it had not been properly validated to be used in local setting.

Overall, public awareness on colorectal cancer, symptoms and concept of cancer screening were relatively good. Majority of studied population were also willing to participate in future cancer screening programs even though asymptomatic. However, not many are willing to pay for the colonoscopy cost and rather wait for several months to get it done freely. In order to make the future colorectal cancer screening programs successful, cancer educations should be promoted to rural populations with more involvement from family physicians and primary care doctors.

\section{Acknowledgements}

We acknowledge the support of Director General of Health Malaysia for granting permission to publish this paper. Our gratitude to Ms Norhafiza, Ms Ertina, Ms Aishah, Ms Wan Muhana and Ms Zainab for their kind helps to distribute and collect the survey questionnaires.

\section{References}

Al-Naggar RA, Bobryshev YV (2013). Knowledge of colorectal cancer screening among young Malaysians. Asian Pac J Cancer Prev, 14, 1969-74.

Delgado-Plasencia L, Lopez-Tomassetti-Fernandez E, Hernandez-Morales A, Torres-Monzon E, GonzalezHermoso F (2009). Willingness to undergo colorectal cancer screening in first-degree relatives of hospitalized patients with colorectal cancer. J Med Screen, 16, 33-38. 
Harewood GC, Murray F, Patchett S, et al (2009). Assessment of colorectal cancer knowledge and patient attitudes towards screening: is Ireland ready to embrace colon cancer screening? Ir J Med Sci, 178, 7-12.

Harmy MY, Norwati D, Norhayati MN, et al (2012). Participation and barriers to colorectal cancer screening in Malaysia. Asian Pac J Cancer Prev, 13, 3983-7.

Hashim SM, Fah TS, Omar K, et al (2011). Knowledge of colorectal cancer among patients presenting with rectal bleeding and its association with delay in seeking medical advice. Asian Pac J Cancer Prev, 12, 2007-11.

Hewitson P, Ward AM, Heneghan C, et al (2011). Primary care endorsement letter and a patient leaflet to improve participation in colorectal cancer screening: results of a factorial randomised trial. Br J Cancer, 105, 475-80.

Hilmi I, Hartono JL, Goh K (2010). Negative perception in those at highest risk --potential challenges in colorectal cancer screening in an urban Asian population. Asian Pac J Cancer Prev, 11, 815-22.

Hlavaty T, Lukac L, Huorka M, Bezayova T, Duris I (2005). Positive family history promotes participation in colorectal cancer screening. Bratisl Lek Listy, 106, 318-23.

Joseph J, Vaughan R, Strand H (2015). Effectiveness of nurseperformed endoscopy in colorectal cancer screening: a systematic review. Gastrointestinal Nursing, 13, 26-33.

Lim GCC, Halimah Y (2004). Second Report of the National Cancer Registry. Cancer Incidence in Malaysia 2003. National Cancer Registry. Kuala Lumpur.

Lim KG (2001). Colorectal cancer. In 'A review of Diseases in Malaysia', Eds Lim KG. Taiping Perak, 91-92.

Loh KW, Majid HA, Dahlui M, Camilla A (2013). Sociodemographic Predictors of Recall and Recognition of Colorectal Cancer Symptoms and Anticipated Delay in Help-Seeking in a Multiethnic Asian Population. Asian Pac J Cancer Prev, 14, 3799-804.

Ma Y, Yang Y, Wang F, Zhang P, Shi C, et al (2013). Obesity and Risk of Colorectal Cancer: A Systematic Review of Prospective Studies. PLoS ONE, 8, 53916.

Office for National Statistics (2014). Cancer Registration Statistic 2012, England. In 'Statistical Bulletin'.

Radzi AHM, Khamizar WKW, Zabedah O, et al (2014). The Second Report of The National Cancer Patient RegistryColorectal Cancer, 2008-2013, Kuala Lumpur, Malaysia.

Singh H, Samadder NJ, Guha S (2014). Flexible sigmoidoscopy for colorectal cancer screening in Canada. Can J Gastroenterol Hepatol, 28, 21-22.

Su TT, Goh JY, Tan J, Muhaimah AR, Pigeneswaren Y, Khairun NS, et al (2013). Level of colorectal cancer awareness: a cross sectional exploratory study among multi-ethnic rural population in Malaysia. BMC cancer, 13, 376.

Sung JJ, Ng CS, Chan FKL, et al (2015). An updated Asia Pacific consensus recommendation for colorectal cancer screening. Gut, 64, 121-32.

Tan YM, Rosmawati M, Ranjeev P, Goh KL (2002). Predictive factors by multivariate analysis for colorectal cancer in Malaysian patients undergoing colonoscopy. J Gastroenterol Hepatol, 17, 281-4.

Tung SY, Wu CS (2000). Risk factors for colorectal adenomas among immediate family members of patients with colorectal cancer in Taiwan - a case control study. Am J Gastroenterol, 95, 3624-8.

Weller DP, Patnick J, McIntosh HM, Dietrich AJ (2009). Uptake in cancer screening programs. Lancet Oncol, 10, 693-9.

Wong MCS, Hirai HW, Luk AKC, et al (2013). The Knowledge of Colorectal Cancer Symptoms and Risk Factors among 10,078 Screening Participants: Are High Risk Individuals More Knowledgeable? PLOS ONE, 8, 60366. 